\title{
Les rêves de la raison
}

Jean-Pierre Dupuy

\section{(2) OpenEdition}

\section{Journals}

Édition électronique

URL : http://journals.openedition.org/ress/337

DOI : 10.4000/ress.337

ISSN : 1663-4446

\section{Éditeur}

Librairie Droz

\section{Édition imprimée}

Date de publication : 1 novembre 2004

Pagination : V-XIII

ISBN : 2-600-00980-9

ISSN : 0048-8046

Référence électronique

Jean-Pierre Dupuy, "Les rêves de la raison», Revue européenne des sciences sociales [En ligne], XLII-130 | 2004, mis en ligne le 03 novembre 2009, consulté le 07 mai 2019. URL : http:// journals.openedition.org/ress/337 ; DOI : 10.4000/ress.337 
Jean-Pierre DUPUY

\section{LES RÊEES DE LA RAISON}

«We have met the Enemy and He is Us.»

Pogo Possum

L'ouvrage que j' ai le plaisir de préfacer réussit le tour de force de présenter, de façon bien plus subtile que tout ce qui a été fait à ce jour, les raisons et les implications du principe de précaution, tout en le remettant à sa juste place, qui est loin d'être aussi grande que tout le bruit médiatique qui l'accompagne le donnerait à penser. En France, les partisans du principe n'auront de cesse qu'il ne figure dans le préambule de la Constitution, acquérant ainsi la même dignité que la déclaration des droits fondamentaux. Les ennemis du principe ne désarmeront pas tant que la science n'aura pas écrasé les prétentions de cet avorton qui croit pouvoir s'opposer à sa glorieuse progression. Après avoir lu ce livre, on se dit que c'est là beaucoup de bruit pour pas grand-chose. Mark Hunyadi, l'organisateur de l'ouvrage, qui, comme le grand Jean-Jacques, a le double avantage d'être philosophe et citoyen de Genève ${ }^{1}$, termine son introduction théorique par ces dures paroles: «Entièrement situé sous le paradigme instrumental du calcul des conséquences, le principe de précaution ne vise qu'à gérer rationnellement les incertitudes que la pensée instrumentale a elle-même produites. [...] Il n'est qu'une aide à la raison instrumentale pour la prévenir de ses propres excès - un correctif au service de la cause qu'elle corrige.» Et, plus terrible encore, cet avertissement: «Le principe de précaution risque de devenir le cache-sexe de l'irréflexion politique.»

Se recrutant pour l'essentiel dans l'establishment scientifique et technocratique, les ennemis dudit principe ne devraient pas se réjouir pour autant. Ils ne se rendent pas compte qu'ils ont trouvé, dans ceux qu'ils prennent pour leurs adversaires, plus scientistes qu'eux. Ces derniers, les promoteurs du principe, ne comprennent d'ailleurs pas dans leur naïveté que la science les rejette, alors qu'ils ont le sentiment, et l'ambition, d'être à son service en la préservant de ses débordements. Or ce livre montre admirablement qu'il existe un objet digne d'être pensé sous le vocable de "précaution», mais qu'on se trompe en cherchant à en faire un principe d'action fondé sur la rationalité instrumentale, alors qu'il s'agit d'un mode de raisonnement pratique. Se distinguant de la déjà pléthorique littéra-

Peu de philosophes en France ont assez de respect pour la science et la technique, et la culture suffisante, pour en faire des objets philosophiques à part entière. L'héritage de Sartre et des intellectuels continue hélas de produire ses effets délétères. Il ne faut pas chercher plus loin les raisons de l'indigence du débat public en France sur ces questions, lequel débat se réduit le plus souvent à l'affrontement féroce d'opinions mal informées ou simplement débiles. 
ture sur le sujet, ce livre restitue les débats sur la précaution dans leur juste contexte. L'idée de flanquer la bonne vieille prévention d'une nouvelle venue, la précaution, ne serait pas venue à l'humanité si celle-ci ne s'était pas constituée en quasi-sujet au moment même où elle prenait conscience que sa survie était en jeu. Comme l'écrit fortement Pascal van Griethuysen, dans sa propre contribution introductive au débat: «L'enjeu de la précaution est d'abord global, et correspond à la survie de l'humanité, menacée par le développement de la technique contemporaine.»

Les auteurs de ce livre ont choisi d'appeler "précaution» cet objet de pensée qu'il faut construire de fond en comble à l'écart de ceux qui, malgré leurs efforts pour faire du neuf, continuent inexorablement de le rabattre sur le calcul des conséquences. Ils ont donc choisi de se battre sur le terrain de ceux-là mêmes qu'ils critiquent. Je n'ai pas eu, quant à moi, ce courage. Jugeant que la cause était perdue, j'ai préféré battre en retraite, et user d'un autre vocable pour désigner ce lieu où la prévention ne suffit plus et où il faut penser, et surtout agir, autrement. Je me suis fixé sur «catastrophisme», tentant de donner ses lettres de noblesse à un terme que les précautionneux raisonnables emploient pour rejeter dans l'enfer de l'irrationnel les disciples du philosophe allemand Hans Jonas. Le choix d'un vocable n'est pas seulement affaire de préférence sémantique, il peut avoir une valeur stratégique décisive, ainsi que le montre avec beaucoup de clairvoyance Barbara Bordogna Petriccione. Traitant de «l'utilisation de la notion de risque dans le débat public sur les biotechnologies », elle souligne qu'à quelques exceptions près, il serait impensable aujourd'hui, au regard du droit international et des principes qui régissent le commerce mondial, de s'opposer à la diffusion d'une innovation technologique pour un autre motif que les «risques» qu'elle est susceptible d'entraîner. Encore faut-il que l'existence de ces risques ait été établie selon des procédures scientifiques et qu'ils portent sur des objets consensuellement valorisés, essentiellement la santé et l'environnement. On ne voit pas que l'on pourrait imposer un moratoire sur des recherches au motif qu'elles altéreraient significativement l'image que nous nous faisons de la personne humaine, ou bien notre rapport à la nature!

Si l'on est sensible à cette évidence que l'évaluation des risques, à laquelle se limite le principe de précaution, n'est que la partie émergée de l'iceberg, se pose la question de savoir, lorsqu'il s'agit d'évaluer normativement une technique nouvelle, s'il vaut mieux, pour attirer l'attention sur les autres effets de cette technique, les appeler «risques » ou choisir un terme différent. Dans le premier cas, on se soumet à l'idéologie régnante pour mieux ruser avec elle, peut-être, mais au prix de faire perdre à la notion de risque toute spécificité.

Placé devant ce dilemme, j'ai pour ma part là aussi choisi de me battre sur un autre terrain. Ce livre montre bien qu'on a beau multiplier et raffiner les distinctions conceptuelles, entre risques avérés, potentiels, hypothétiques ou conjecturés, reconnus ou non reconnus, personne à la fin n'y comprend plus goutte ${ }^{2}$ et,

Au moment où j'écris ces lignes, le projet français de loi constitutionnelle relatif à la Charte de l'environnement présenté par le Premier ministre bat tous les records de confusion conceptuelle. Il stipule que le principe de précaution «s'applique uniquement à un dommage dont la réalisation est incertaine en l'état des connaissances scientifiques et doit être distingué de l'action de prévention qui vise à faire face à un risque certain de dommage.» L'expression «risque certain de 
surtout, on conforte ainsi l'économisme ambiant qui ne sait évaluer qu'en balançant des coûts (sous forme de risques) et des avantages. Or, comme le montre Pascal van Griethuysen, «il ne peut être question de justifier une politique de développement technologique sur la base d'une mise à niveau des avantages et des inconvénients du développement technologique.»

Pour manifester aux auteurs de ce livre ma gratitude pour l'honneur qu'ils me font en m'offrant de préfacer leurs travaux, je voudrais ici présenter quelques réflexions qui vont exactement dans ce sens. Leur objet en est les nanotechnologies, ou plus précisément la «convergence» entre les nanotechnologies, les biotechnologies et les technologies de l'information sous l'égide idéologique des sciences cognitives ${ }^{3}$.

Très rapidement, j'ai compris le piège que la notion de risque tendait à celui qui veut procéder à une évaluation normative du programme technologique en question. Le premier des risques à être invoqué dans tous les rapports officiels est celui de «ne pas être dans la course ». Effectivement, et pour de solides raisons, les gouvernements des nations et des régions du globe sont terrorisés à l'idée de perdre une course industrielle, économique et militaire très rapide où vont se jouer les emplois, la croissance, mais aussi les capacités de défense de demain. D'emblée, la question est entendue et le philosophe critique n'a plus qu'à aller se réfugier dans son poêle.

On ne peut pas dire que les promoteurs des nanotechnologies ignorent complètement la question des risques, au sens plus ordinaire du terme. Voici par exemple comment la plaquette que le Ministère français de la recherche et des nouvelles technologies vient de publier pour mieux faire connaître le «nanomonde» aborde la question: «Les enjeux sociétaux des nanotechnologies sont importants. La perspective de manipuler la matière à l'échelle moléculaire et d'interférer avec le monde du vivant pose bien évidemment des questions d'éthique. [...] Tout progrès scientifique comporte un risque, mais les risques des nanosciences, qui peuvent dans une certaine mesure être maîtrisés, sont à mettre en balance avec les immenses avantages qu'elles apportent, dans le domaine de la santé et de l'environnement notamment. [...] [Il faut faire] confiance à l'intelligence de chacun pour conclure que les avantages de ces applications l'emportent de loin sur les inconvénients, qui peuvent être maîtrisés. » On ne saurait mieux illustrer cette réduction de l'évaluation sociale et éthique à la seule problématique du calcul coûts-avantages et à l'analyse des risques. On ne saurait avouer avec plus de franchise que, la balance des risques et des avantages étant radicalement impossible à

dommage » réussit le tour de force d'être à la fois pléonastique (le risque implique le dommage) et contradictoire (le « risque certain » fait partie de ces objets introuvables qui servent à définir en mathématiques l'ensemble vide !) En bon français, on écrirait tout simplement «dommage certain», mais alors la définition de la prévention devient absurde: elle confond le fait épistémique qu'une probabilité est connue («risque») et le fait ontologique que cette probabilité est égale à un («certain»). Je n'ai pas d'opinion arrêtée sur la question de savoir si le principe de précaution doit ou non figurer dans la Constitution - la question ne mérite sans doute pas qu'on se batte pour elle, quel que soit le camp que l'on choisisse! - mais je sais en tout cas qu'il serait honteux pour le pays de Descartes qu'une telle purée mentale accède à la place suprême.

J'utilise l'expression de convergence en référence à l'initiative de la National Science Foundation (NSF) qui a pour titre «Converging Technologies for Improving Human Performances » (2002). Rapport disponible sur la Toile à http://www.wtec.org/ConvergingTechnologies/ 
effectuer, c'est par pétition de principe que l'on déclare que les seconds l'emportent «immensément» sur les premiers. On ne saurait insulter l'intelligence du public avec plus d'insolence.

Du côté des opposants, le tableau n'est guère plus brillant. Les risques les plus extrêmes sont brandis et bientôt mis en scène par les medias, la littérature et le film. On imagine une autoréplication sauvage de nanomachines à la suite d'un accident de programmation ou du fait d'un acte terroriste. Tout ou partie de la biosphère serait alors détruite par épuisement du carbone nécessaire à l'autoreproduction des nano-engins en question. Ce risque ne peut vraiment effrayer que celui qui croit à la possibilité de telles machines. Il suffit de nier cette possibilité pour écarter le pseudo-risque d'un haussement d'épaules.

Pendant que les participants à ce dialogue de sourd s'invectivent au sujet de la réalité des risques et des avantages ou pour savoir lesquels l'emporteront dans la balance du calcul économique, personne ne réfléchit à des enjeux ou des effets qui, bien que plus cachés, n'en sont pas moins fondamentaux. De ces effets, l'analyse en termes de risques n'a rien à dire. Pour qu'il y ait risque trois éléments doivent être présents: a) une éventualité de dommage, affectée normativement d'un signe moins; b) un degré de vraisemblance (pas nécessairement une probabilité) assigné en principe à l'occurrence de ce dommage; c) une population d'individus touchés potentiellement par le dommage et dont les «utilités» (ou «satisfactions», ou «ophélimités» etc.) servent d'étalon pour l'appréciation du dommage. Le débat sur le principe de précaution a introduit une distinction d'ordre épistémique, à savoir le type de connaissance que les acteurs ont ou n'ont pas du degré de vraisemblance de l'occurrence du dommage, par exemple sous la forme de probabilités objectives. Je crains que ce débat n'ait brouillé les pistes en détournant de l'essentiel. L'essentiel, ce sont ces effets qui ne sont pas des risques, en ce qu'ils ne satisfont aucune des trois conditions que je viens de rappeler. Lorsque la National Science Foundation dit des nanotechnologies qu'elles vont «entraîner un changement de civilisation », bien malin serait celui qui s'aventurerait à mettre un signe, plus ou moins, devant cette éventualité, qui se prononcerait sur son degré de vraisemblance ou qui en évaluerait les conséquences en additionnant les différentiels d'«utilités» sur toute la population.

Pour échapper au vocabulaire (et à la pensée) économique, j'ai parlé des effets du programme nanotechnologique, et en ai proposé la typologie suivante.

\section{LES EFFETS SUR LES RELATIONS DE DOMINATION (EFFETS DE POUVOIR)}

Nous savons bien que ces effets sont considérables et que ce sont eux qui, au fond, mobilisent l'opinion et expliquent l'essentiel de ses rejets. Comme tous les acteurs - y compris les opposants -, ont, certes inégalement, des enjeux de pouvoir, un déplacement, comme on dirait en psychanalyse, a souvent lieu, et le débat en vient à porter uniquement sur les risques. Ce qui en vérité est en jeu va de l'appropriation par un tout petit nombre de firmes des conditions de production et de reproduction de la vie à la domination qu'exercent la science et la technique sur des populations qui dans leur immense majorité n'ont pas accès à la culture scientifique et technique; de l'humiliation que les scientifiques ressentent à devoir se 
livrer à des opérations publicitaires ou de relations publiques pour conquérir une «acceptabilité » de plus en plus évanescente à la colère de ceux qui n'ont plus la maîtrise de ce qu'ils mangent; de l'approfondissement des inégalités mondiales aux nouvelles pauvretés engendrées par le monopole qu'exercent les techniques nouvelles sur des actes ou des relations qui traditionnellement échappaient à la technique; etc.

\section{LES EFFETS SUR LE RAPPORT À LA NATURE (EFFETS ONTOLOGIQUES)}

Le débat actuel sur la transformation du rapport à la nature provoquée par les techniques nouvelles se présente ainsi. D'un côté, l'écologie profonde qui fait de la nature un modèle immuable d'équilibre et d'harmonie, et de l'homme un prédateur irresponsable et dangereux; de l'autre, le projet humaniste moderne d'arracher l'homme à la nature et de le rendre maître et possesseur du monde et de lui-même. Dans un cas la «transgression» est vilipendée, dans l'autre elle est revendiquée.

Le débat ainsi engagé passe selon moi à côté de l'essentiel. En arrière-fond de tout «paradigme» scientifique et technique, il y a ce que Karl Popper appelait un «programme métaphysique de recherches»- ensemble non testable de propositions que l'on tient pour vraies sans chercher à les remettre en cause, cadre théorique qui limite le type de questions que l'on pose mais aussi qui en donne l'inspiration première. Le programme métaphysique de recherches des nanotechnologies tient dans les deux mots d'ordre suivants: a) Il faut viser à naturaliser l'esprit et la vie pour qu'ils retrouvent leur place au sein de la nature qui les a engendrés; b) Cette naturalisation passe par une mécanisation et une artificialisation, tant de la nature que de la vie et de l'esprit. Si les nanotechnologies ambitionnent de prendre le relais de la nature et de la vie, ce n'est que parce qu'elles ont auparavant complètement redéfini ces dernières à leur image. L'expression «nature artificielle» n'est désormais plus un oxymore.

Une fois admise une telle vision du monde, il n'y a qu'un pas pour en arriver à former le projet de se rendre maître de ces machines informationnelles ou algorithmiques, d'abord en les simulant et en les reproduisant (naissance de l'intelligence, puis de la vie artificielles), ensuite en intervenant sur elles à la manière de l'ingénieur (biotechnologies, technologies cognitives, etc.). Le problème n'est plus de savoir jusqu'à quel point on peut ou on doit «transgresser» la nature. Le problème, c'est que la notion même de transgression est sur le point de perdre tout sens. L'homme ne rencontrera jamais plus qu'un monde à l'image de ses propres créations artificielles.

\section{LES EFFETS SUR LE RAPPORT À LA CONNAISSANCE (EFFETS ÉPISTÉMIQUES)}

A l'aube des temps modernes, Jean-Baptiste Vico formula dans des termes célèbres le postulat de la «nouvelle science» (1725): «Verum et factum convertuntur» (Ce qui est vrai et ce que l'on fait sont convertibles). Nous ne pouvons 
connaître rationnellement que ce dont nous sommes la cause, que ce que nous avons fabriqué. Par ordre décroissant de perfection de la connaissance, les mathématiques, selon ce critère, étaient classées en premier, suivies non par les sciences de la nature, mais par les sciences morales et politiques. Cependant, la science de la nature elle-même devait être dès les commencements orientée par la conviction qu'on ne peut connaître qu'en faisant. L'insistance sur le comment des processus plutôt que sur l'être des choses s'explique ainsi, mais aussi et surtout le rôle considérable dévolu à l'expérimentation et à la modélisation par la science.

Avec les nanotechnologies, le verum factum devrait trouver son aboutissement ultime. Ce n'est plus seulement en faisant des expériences sur elle, ce n'est plus seulement en la modélisant, que les hommes connaîtront la nature. C'est littéralement en la re-faisant. Mais, du coup, ce n'est plus la nature qu'ils connaîtront, mais ce qu'ils auront fait. Ou plutôt, c'est l'idée même de nature, donc de donné extérieur à soi, qui apparaîtra comme dépassée. La distinction même entre connaître et faire perdra tout son sens, de même que celle qui sépare encore aujourd'hui le savant de l'ingénieur ${ }^{4}$.

\section{LES EFFETS SUR LA POSSIBILITÉ MÊME DE L'ÉTHIQUE (EFFETS ÉTHIQUES)}

Les nanotechnologies ouvrent un continent immense que l'homme va devoir normer s'il veut leur donner sens et finalité. Il faudra que le sujet humain recoure à un surcroît de volonté et de conscience pour déterminer, non pas ce qu'il peut faire, mais bien ce qu'il doit faire. Il y faudra toute une éthique, infiniment plus exigeante que celle qui, aujourd'hui, se met lentement en place pour contenir le rythme et les dérives des biotechnologies. Qui dit «éthique», «conscience», «volonté» dit le triomphe du sujet. Mais que signifie ce triomphe dans une conception du monde qui traite la nature, y compris l'homme, comme une machine computationnelle ? Cet homme qui s'est ainsi fait machine, au nom de quoi ou de qui va-t-il exercer son immense pouvoir sur la nature et sur lui-même? Au nom du mécanisme aveugle auquel il s'identifie? Au nom d'un sens dont il prétend qu'il n'est qu'apparence ou phénomène? Sa volonté et ses choix ne peuvent qu'être suspendus dans le vide. L'élargissement sans limites du champ de l'éthique menace à terme la possibilité même de l'éthique.

\section{LES EFFETS SUR LES CATÉGORIES (EFFETS MÉTAPHYSIQUES)}

On peut tout à la fois considérer que les métaphores mécanistes et informationnelles sur lesquelles se sont bâties tant les sciences cognitives que la biologie moléculaire sont scientifiquement et philosophiquement fausses et concéder qu'elles nous donnent une puissance d'agir et une maîtrise radicalement inédites

On voit déjà aujourd'hui avec les seules biotechnologies que la distinction entre découverte et invention, sur laquelle repose le droit des brevets, est de plus en plus délicate à tracer, ainsi que l'attestent les débats sur la brevetabilité du vivant. 
sur le donné naturel et vivant. Si tel est le cas, les succès mêmes que remporteront ces nouvelles technologies rendront les représentations mécanistes et informationnelles de la nature et de la vie incontestables et nul ne pourra plus voir qu'elles sont illusoires. Il n'est pas exagéré de parler d'effets métaphysiques.

L'effet le plus troublant est sans conteste le brouillage des distinctions catégorielles au moyen desquelles l'humanité, depuis qu'elle existe, s'est toujours repérée dans le monde. Le naturel non vivant, le vivant et l'artefact sont en bonne voie de fusionner.

Ces effets sont graves et ce n'est pas parce qu'on ne peut dire simplement s'ils sont «bons» ou «mauvais», encore moins les quantifier ou leur affecter des probabilités d'occurrence, qu'on doit les négliger. A se limiter à la question des risques, on regarderait là où il y a de la lumière - tout en sachant que ce que l'on cherche est ailleurs. Je ne puis une fois de plus que souscrire aux fortes paroles de Mark Hunyadi : «il se pourrait que petit à petit, le principe de précaution s'autonomise à son tour, hypnotisant la rationalité politique au point de faire de l'évaluation des risques le seul critère de décision légitime, comme si les risques étaient le seul aspect sous lequel il faille évaluer une situation d'incertitude. Nous connaissons la «société du risque », mais celle-ci a tôt fait de produire une idéologie du risque qui peut se donner la bonne conscience du principe de précaution tout en soustrayant les décideurs à leur responsabilité politique - comme si évaluer, calculer, soupeser des risques était l'ultima ratio, le critère ultime de la décision politique. Il y a donc un usage scientiste du principe de précaution en politique qui risque d'en faire, plutôt que le contrepoids, le symptôme de la civilisation technologique qui a été son contexte d'émergence.»

Une gravure terrifiante de Goya porte le titre «El sueño de la razón produce monstruos $»^{5}$. Titre ambigu au possible, puisque le mot «sueño», en espagnol, signifie indifféremment «sommeil» ou «rêve». En français, on traduit généralement par «Le sommeil de la raison produit des monstres», et l'on comprend: «Lorsque la raison est en sommeil, c'est-à-dire mise entre parenthèses, alors l'imagination produit des monstres.» C'est peut-être le sens voulu par Goya. L'anglais cependant traduit: «The dream of reason...» De façon beaucoup plus intéressante, le sens devient: «Les rêves de la raison engendrent des monstres ». C'est la raison elle-même, et non son absence, qui a cette capacité de faire advenir par ses rêves des choses monstrueuses.

Je crois que tout mon rapport à la raison est pris dans cette ambiguïté. Mark Hunyadi me présente comme l'un des champions de l'école «catastrophiste» mais aussi comme un «héraut de la rationalité». Je m'acharnerais à pousser la «raison calculatrice» jusque dans ses ultimes frontières de façon à trouver une solution rationnelle au problème qui est le nôtre aujourd'hui - sauver l'humanité contre elle-même.

Héraut, je le suis peut-être, mais doublé d'un traître. La critique de Mark Hunyadi me poussant dans mes retranchements, je me vois obligé de faire un aveu. Je lui sais gré de m'empêcher de me dérober.

\footnotetext{
http://www.bne.es/Goya/c75.html
} 
Certes, je ne partage pas le jugement sans appel qui semble être celui de tous les auteurs de ce livre au sujet de ce qu'ils appellent, après Max Weber, la rationalité instrumentale, ou encore, comme Heidegger, la raison calculatrice. Ou plutôt, je demande qu'avant de condamner, on examine précisément l'identité précise de l'accusé et ce dont il est capable ${ }^{6}$. Ce sont les philosophes rationalistes euxmêmes ${ }^{7}$ qui ont montré que la notion de rationalité instrumentale [Zweckrationalität] n'avait en vérité en elle-même aucune consistance, car non seulement on ne peut la définir indépendamment d'une rationalité des fins ou des valeurs [Wertrationalität], mais elle est complètement subordonnée à cette dernière ${ }^{8}$. Plus profondément encore, ils ont établi que la rationalité telle que l'entend la théorie du choix rationnel était en quelque sorte capable de s'auto-transcender et d'engendrer une raison qui la dépasse, une raison capable d'énoncer des impératifs catégoriques, et non seulement hypothétiques.

Ce n'est cependant pas dans le cadre de cette raison qui reste «calculatrice» que j'ai tenté, après Hans Jonas, de trouver une réponse métaphysique à la crise d'une humanité qui se révèle à elle-même au moment où sa survie est en jeu. Comme le philosophe allemand, je suis parti d'une aporie éthique: aujourd'hui, la seule éthique qui vaille est une éthique conséquentialiste, tant les effets de nos actions sur le monde sont devenus considérables. Mais la mise en œuvre de cette éthique est impossible, et cela pour les raisons mêmes qui rendent le conséquentialisme incontournable. Notre puissance d'agir est telle que nous ne savons plus ce que nous faisons alors même que nous devons, plus que jamais, le savoir. L'impasse est totale.

Ce n'est donc certainement pas dans un calcul des conséquences poussé jusqu'à je ne sais quelle extrémité que j'ai cherché une issue. Cette voie est complètement bouchée. L'issue, je crois l'avoir trouvée dans un tour de force, dans lequel on est libre de voir un tour de passe-passe. Il s'agit de clore le temps, cet ouvert par excellence, et de contempler et juger notre présent depuis cette clôture. Puisque notre problème est celui de la mort de l'humain, c'est cette

Certaines des contributions à ce livre me semblent ne pas vraiment satisfaire cette condition. Il est par exemple inexact d'affirmer que la théorie du choix rationnel implique une «connaissance parfaite des probabilités d'occurrence». La théorie du choix rationnel telle qu'elle a été axiomatisée et formalisée par Leonard Savage ne présuppose en rien que les probabilités soient connues, ni d'ailleurs inconnues. Les probabilités ne font l'objet d'aucune connaissance pour la bonne raison qu'elles ne se réfèrent plus à des régularités de la nature (sous la forme de fréquences d'occurrence) mais à la cohérence des choix opérés par l'agent - cette cohérence étant définie par la satisfaction des axiomes de la théorie. C'est pourquoi ces probabilités sont dites subjectives. C'est précisément cet affranchissement de la théorie par rapport à toute idée de connaissance qui explique, d'une part, sa très grande généralité (que Savage lui-même prenait pour universalité) et, d'autre part, qu'il soit si difficile de s'en déprendre. La plupart des travaux réalisés par des économistes sous le label du principe de précaution continuent de se situer dans le cadre de la théorie de Savage (dite encore Bayésianisme) ou de ses nombreux avatars.

Je pense en particulier aux travaux de David Gauthier et de la très regrettée Jean Hampton.

Pour s'en convaincre, il suffit à vrai dire d'examiner les axiomes de Savage ou de von Neumann: ils portent tous sur ce qu'il est rationnel de désirer. Ainsi de l'»axiome de la chose certaine» de Savage (Sure Thing Principle): si je préfère l'option $a$ à l'option $b$ si l'état du monde est X, et que je préfère également $a$ à $b$ si l'état du monde n'est pas X, alors je dois préférer $a$ à $b$ même si je ne connais pas l'état du monde. Je choisis cet exemple car il illustre la remarque que j'ai faite plus haut au sujet de l'indifférence à l'état de connaissance. 
clôture-là que j'ai prise comme point de vue. Or la fin de l'humanité, comme celle d'un individu, transforme la vie, et l'histoire, en destin. Si j'ai établi quelque chose, c'est que nous ne pouvons éviter de poser la question du destin et du sens de l'humanité comme transcendant chacun des moments de son histoire. La clôture du temps fait immédiatement surgir une dimension de verticalité. On est très loin du calcul des conséquences, qui présuppose des futurs possibles, disposés en arborescence, en une pure immanence.

Mark Hunyadi se demande à mon sujet si la logique, fût-elle métaphysique, peut à elle seule avoir force motivationnelle. La réponse est évidemment négative. Si la logique m'a servi à quoi que ce soit, c'est à montrer qu'on ne peut faire l'économie des grandes questions qui agitent les humains depuis l'origine des temps et auxquelles les religions de tout temps ont donné des réponses. La «rationalité pure» - mais cela est un oxymore - en est bien incapable. Mais c'est aussi le cas de la «rationalité procédurale» dont certains voient la quintessence dans le principe de précaution. L'appel à la démocratie est trop souvent l'alibi du renoncement à poser la question de la transcendance. On ne détermine pas le sens de l'existence par un vote.

Pour le dire autrement, la question de la rationalité ne m'intéresse que du point de vue anthropologique. Le même Max Weber qui nous a donné la rationalité instrumentale a montré que celle-ci était fille du religieux. Comme le dit Michel Serres, le mythe est dense dans la science, et la science dans le mythe. Le sacré, avec ses deux faces, la terreur et la vénération, est dense dans la raison. Si celle-ci peut se transcender elle-même, c'est qu'elle contient déjà en elle la transcendance. Nous ne trouverons pas d'issue à la crise sans un principe d'autolimitation. Il n'y a pas d'autolimitation sans auto-transcendance. Mon aveu, c'est que je crois qu'il n'y a pas d'auto-transcendance sans véritable transcendance.

Cependant, ce n'est pas le lieu pour moi de défendre mes thèses, mais de dire simplement mon admiration pour un travail collectif exemplaire, qui devrait faire date dans le débat qui ne fait que s'ouvrir sur les conditions de survie de l'humanité. 